\title{
REFLEXIONES A LOS PLANES DE ESTUDIO DE BIOLOGIA
}

\section{REFLECTIONS ON THE BIOLOGY CURRICULUM}

\section{AUTORES}

José María Barrasa: Biólogo y Profesor Titular del Departamento de Biología Vegetal jose.maria@arrakis.es

\section{CURRÍCULUM VITAE}

Profesor en la Facultad de Biología. Universidad de Alcalá de Henares.

\section{RESUMEN}

El artículo que presentamos, escrito por el profesor del Departamento de Biología Vegetal, D. José María Barrasa, nos ha servido de introducción al debate de la tertulia de la GRUA, celebrada el pasado 20 de Enero. Está pensado como reflexión sobre la contrarreforma de los planes de estudio de la Licenciatura de Biología. Sin embargo, sustituyendo la palabra Biología por la correspondiente a otra rama de la Ciencia, tiene completa validez en cualquiera de los estudios que se imparten en la Universidad de Alcalá, e incluso en muchas otras Universidades españolas.

Como todos sabemos, actualmente se está elaborando (otra vez) el nuevo plan de estudios en Biología. Me consta el gran esfuerzo y la buena voluntad que desde el Decanato de Ciencias y su Comisión delegada se está realizando para la consecución de los nuevos planteamientos docentes en Biología. Sin embargo, como Biólogo y Profesor en la licenciatura de Biología desde hace casi veinte años, me gustaría hacer unas reflexiones a título personal. Tal vez, y pido disculpas por ello, estas reflexiones estén algo desordenadas, sean subjetivas y, probablemente, también parciales; pero están realizadas con el mayor espíritu de cooperación. 


\section{PALABRAS CLAVE}

Reflexiones - Planes de estudio - Biología

\section{ABSTRACT}

The present article written by Professor, Department of Plant Biology, D. José María Barrasa, has served as an introduction to the discussion of the social gathering of the crane, held last January 20. It is intended as a reflection on the counter of the curriculum for the Bachelor of Biology. However, replacing the word for Biology from the other branch of science, has full validity in any of the courses taught at the University of Alcalá, and even in many other Spanish universities.

As we all know, is currently under development (again) the new biology curriculum. I know the great effort and goodwill from the Dean of Sciences and its Executive Committee is making to the achievement of new teaching approaches in biology. However, as a biologist and professor at the undergraduate biology for nearly twenty years, I would like to make some personal reflections. Perhaps, and I apologize for that, these reflections are a little messy, are subjective and probably also partial, but are made with the greatest spirit of cooperation.

\section{KEY WORDS}

Reflections - Curriculum - Biology

\section{ÍNDICE}

1. Calidad de la docencia

2. Créditos teóricos y prácticos

3. Perfil formativo 


\section{Optatividad y libre elección}

5. Especialidades

6. Horario

El artículo que presentamos, escrito por el profesor del Departamento de Biología Vegetal, D. José María Barrasa, nos ha servido de introducción al debate de la tertulia de la GRUA, celebrada el pasado 20 de Enero. Está pensado como reflexión sobre la contrarreforma de los planes de estudio de la Licenciatura de Biología. Sin embargo, sustituyendo la palabra Biología por la correspondiente a otra rama de la Ciencia, tiene completa validez en cualquiera de los estudios que se imparten en la Universidad de Alcalá, e incluso en muchas otras Universidades españolas.

Como todos sabemos, actualmente se está elaborando (otra vez) el nuevo plan de estudios en Biología. Me consta el gran esfuerzo y la buena voluntad que desde el Decanato de Ciencias y su Comisión delegada se está realizando para la consecución de los nuevos planteamientos docentes en Biología. Sin embargo, como Biólogo y Profesor en la licenciatura de Biología desde hace casi veinte años, me gustaría hacer unas reflexiones a título personal. Tal vez, y pido disculpas por ello, estas reflexiones estén algo desordenadas, sean subjetivas y, probablemente, también parciales; pero están realizadas con el mayor espíritu de cooperación.

\section{Calidad de la docencia}

Es un lugar común que cuando se habla de la calidad de docencia se aluda frecuentemente a la carencia de medios económicos. Es evidente que la mejora de los medios materiales, un mayor apoyo para infraestructura docente y subvenciones 
económicas más elevadas son, sin duda alguna, elementos valiosos para mejorar la calidad de la enseñanza en cada departamento.

Pero un buen plan de estudios no se obtiene sólo con esto. Las ayudas económicas, aunque necesarias y bien recibidas, no deben llegar solas. Las subvenciones deben llegar además de y nunca en lugar de un plan coherente y de unos profesores preparados y en disposición de ofrecer sus mejores dotes docentes. Existen países con recursos económicos infinitamente inferiores a los nuestros $\mathrm{y}$, según las encuestas, son los primeros en cuanto a calidad docente se refiere. A buen seguro que la causa de ese éxito se debe a una adecuada planificación docente y a una buena formación de su profesorado, sin olvidar probablemente también, un ambiente social propicio.

La elaboración de un plan de estudios coherente es, metodológicamente, un proceso parecido a construir una buena casa. Todo debe hacerse siguiendo un orden lógico ya que es imposible levantar los muros sin, por ejemplo, poner antes unos buenos cimientos. Sobre ellos habrá entonces que poner los pilares, las vigas y los muros maestros. Después vendrán los tabiques interiores, las cubiertas, el tejado y las conducciones. Para finalizar habrá que hacer los remates y poner los detalles, los complementos (sanitarios, mobiliario etc.) y los adornos (cuadros, cortinas, etc.).

Desgraciadamente, el actual plan de estudios de Biología (y me temo que también el que se está elaborando), más bien parece una mezcla caótica de tuberías, cables, puertas, grifos..., mucho más parecido a una chabola que a un buen edificio. Causas de este caos son, a mi modo de ver, la excesiva optatividad, libre elección y libre configuración, dejadas también al libre albedrío de alumnos carentes aún de la necesaria formación y madurez. Ello conduce al despiste generalizado y a la enorme confusión de conceptos y conocimientos que se observa entre los alumnos de nuestra licenciatura. 
Naturalmente, los profesores tampoco estamos exentos de culpabilidad ni somos un dechado de virtudes. En general entre el profesorado falta motivación, afán de superación, dedicación etc. Culpa importante de este desánimo la tiene el hecho de que desgraciadamente la buena labor docente no se premia, es más, se dificulta y se obstaculiza.

Todos sabemos que para que la docencia en cualquier área científica, y por lo tanto también en Biología, sea útil y práctica se requiere que el alumno reciba información clara, concisa y, al menos, medianamente actualizada. Para ello el profesor debe llevar a cabo una laboriosa tarea que consiste en la continua búsqueda, lectura, análisis y síntesis de las publicaciones más recientes relativas a la materia que imparte. Esta "puesta a punto" del profesor requiere muchas horas de estudio, y dinero para la adquisición y recopilación de toda la información bibliográfica necesaria.

Pero, volviendo al comentario realizado anteriormente: ¿de qué manera se obstaculiza (y casi se castiga) la docencia? Como hemos visto, "estar al día" para impartir una buena docencia requiere muchas horas de estudio, lectura y sacrificio personal por parte del profesor. Este trabajo personal no está reconocido ya que los tramos de docencia o quinquenios se conceden automáticamente a todos los profesores cada cinco años hagamos lo que hagamos desde el punto de vista docente. Sin embargo, no sucede lo mismo con los tramos de investigación o sexenios. El Ministerio de Educación, mediante la actuación de su Comisión Evaluadora de la Actividad Investigadora, otorga o deniega los tramos de investigación evaluando (sin entrar aquí a discutir la evaluación en sí misma) la calidad de la investigación llevada a cabo por el profesor durante los seis últimos años. Ante la posibilidad real de que el sexenio sea denegado, el profesor se ve forzado a utilizar su tiempo en 
aquella actividad en la que su trabajo y esfuerzo personal va a ser, de alguna manera, reconocido y premiado.

La docencia queda así abandonada. No merece la pena estar al día para impartir una buena docencia pero si para llevar a cabo una buena investigación. ¿Por qué se coloca al profesor ante esta tesitura y se le somete a tal presión? ¿Qué postura altruista o actitud casi heroica debe mostrar el profesor para que trabaje y prepare sus clases a costa de lo que en realidad y a la hora de la verdad se le va a valorar? ¿Qué deben hacer los profesores con auténtica vocación docente para no desmoralizarse ante tal situación?

Bien mirado, el trabajo dedicado a la docencia es, en realidad, muy similar al que desarrollamos con nuestra labor investigadora. Todos sabemos que para poder publicar los resultados de la investigación en revistas de impacto científico, es necesario estar "al día" en el tema que nos atañe. Esta "puesta a punto" es imprescindible para poder escribir con rigor buena parte de las diferentes secciones de un trabajo científico (introducción, material y métodos, discusión y conclusiones). ¿No deberíamos hacer algo muy parecido cuando impartimos los diferentes temas de nuestra asignatura? Necesitamos primero hacer una introducción al tema que se va a tratar. A veces, hablamos al alumno (o deberíamos hablarle) del método o métodos que se han seguido para su estudio. A continuación desarrollamos y exponemos el estado actual de conocimientos sobre el tema que se trata (equivaldría a la presentación de los resultados de nuestra investigación). Finalmente comentamos (o deberíamos comentar) lo que se desconoce y aún falta por conocer sobre el tema (sería una especie de discusión). Incluso después de explicado el tema proporcionamos la bibliografía sobre la que se ha obtenido la información (la bibliografía citada). 
Desde este punto de vista no existe tanta distancia entre docencia e investigación, e incluso cabe preguntarse si no podría, por lo tanto, existir una investigación enfocada a la docencia.

Por otro lado, y en no pocas ocasiones, se dice que sólo aquel que investiga ya está actualizado en conocimientos y preparación docente. Esto es sencillamente falso. Por un lado, no parece que existan datos que demuestren tal aseveración. Por otro lado, el investigador actual se encuentra abocado a una vía extremadamente reduccionista, en la que cada vez sabe más.....de menos.

Es perfectamente lógico que el profesor no pueda profundizar en todos los campos tanto como en el de su propia especialidad, pero está obligado a conocer las innovaciones y avances de su asignatura, al menos a nivel divulgativo. Somos conscientes que el término "divulgación" goza de mala fama en el ámbito universitario ya que está tildado de poco científico. Sin embargo, muchas veces se argumenta también que hay que realizar un acercamiento de la Universidad al resto de la sociedad. ¿No sería ésta una de las mejores maneras de hacerlo? ¿Por qué no plantearnos de una vez las preguntas verdaderamente importantes y relevantes para mejorar la calidad de la docencia?

\section{Créditos teóricos y prácticos}

Un aspecto importante a la hora de elaborar el plan de estudios de Biología, ya que se trata de una de las licenciaturas llamadas experimentales, será el correcto equilibrio entre los créditos teóricos y prácticos.

Actualmente se observa que en algunas disciplinas de la licenciatura, la docencia se encuentra muy sesgada hacia la parte práctica, quedando la parte teórica reducida a unos pocos créditos de escaso o nulo valor formativo y cuya existencia es meramente 
testimonial. Ante esta situación el alumno aprende, en el mejor de los casos, sólo técnicas (a veces tan concretas que rara vez tendrá ocasión de ponerlas en práctica durante su vida profesional), pero nunca aprenderá un método y menos aún unas bases teóricas, imprescindibles para comprender cualquier aspecto de la ciencia.

Por mi relación laboral con algunos centros de investigación y empresas relacionadas de una u otra manera con la Biología, he tenido ocasión de comprobar, que el modelo de formación de personal que demandan ese tipo de instituciones, es aquel que ofrece personas provistas de una buena formación integral, capaces de aprender con rapidez esa técnica concreta, o el manejo de aquel aparato particular que dicha empresa o centro de investigación necesita para llevar a cabo su producción o su investigación. En la mayoría de los casos el conocimiento de esa(s) técnica(s) concreta(s) se adquiere sobre la marcha, unas veces mediante las indicaciones cotidianas del personal existente, más experimentado, y en otros, incluso mediante la impartición de breves cursillos que algunos centros y empresas disponen para la formación concreta y especializada del nuevo personal contratado.

Un futuro Plan de estudios de Biología debería evitar el exceso de actividades didácticas de tipo procedimental, es decir aquellas relacionadas con la manipulación y el trabajo manual y experimental, tanto de laboratorio como de campo. Un exceso de créditos prácticos puede conducir a situaciones en las que las prácticas queden reducidas simplemente a apretar un botón o una tecla (en el caso de las disciplinas biológicas de laboratorio) o a convertirse en una agradable excursión eco-turística, tanto para el alumno como para el profesor (en el caso de las disciplinas de campo). Igualmente, las prácticas de laboratorio de disciplinas más relacionadas con la diversidad biológica, pueden convertirse en una plácida contemplación visual (como si se tratara de una visita al museo de Ciencias Naturales) de una serie de organismos disecados o conservados en formol. En concreto y en lo que personalmente me resulta más cercano, en los últimos años he podido constatar con pena cómo en éste 
tipo de prácticas no se incide en que el alumno aprenda a distinguir y manejar los caracteres morfológicos de un ser vivo que, en definitiva, es lo que les permitirá después reconocer su identidad a nivel de especie, género, familia, etc. No se incide tampoco en el uso de claves de identificación, tan útiles y prácticas para el desarrollo de la capacidad de observación del futuro biólogo a la hora de analizar un ser vivo. No se incide en definitiva, en que el alumno "caiga en la cuenta", al menos de manera intuitiva, ya que de modo real es imposible por razones obvias, de la extraordinaria diversidad de especies que albergan la mayoría de los grupos biológicos que existen en la naturaleza. En ocasiones, he visto impartir prácticas cuyo único objetivo era el reconocimiento (por supuesto sin el empleo de claves de identificación) de sólo aquellas especies que ¡confieren paisajej. Es decir "las grandes". Como si ser grande tuviera una importancia capital desde el punto de vista biológico o, en este caso, taxonómico.

Vemos así, que sólo se enseña una técnica (y a veces nefasta), pero no un método ni un criterio científico. Parece como si los planes de estudio primasen el entretenimiento en lugar del estudio serio que permita al alumno adquirir la capacidad de analizar, relacionar, sintetizar, concluir y, en definitiva, comprender los fenómenos biológicos del mundo que nos rodea. Nuestros alumnos sabrán "hacer cosas concretas" pero difícilmente podrán plantearse un problema científico o realizar una síntesis. Es cierto que habrá excepciones que confirmen la regla y siempre existirán alumnos capaces de llegar por sí solos a la comprensión científica a partir de la cantidad de datos proporcionados a lo largo de la licenciatura, pero siempre les llevará mucho tiempo y un largo proceso de maduración.

Por todo ello, cada vez es más raro encontrar alumnos y profesores capaces de diferenciar lo que es método y lo que son técnicas, o lo que es lo mismo, cuál es la estrategia del trabajo científico y cuáles sus tácticas, así como el contexto y uso de cada uno de ellos. Esto es el resultado de haber escorado la docencia del lado de la 
tecnología en lugar del lado de la ciencia, se dice que "para atender las demandas prácticas y sociales". No cabe duda que hay que explicar la tecnología en las clases (aún a sabiendas de que puede quedar enseguida obsoleta dados los tiempos que corren) pero hay que explicarla siempre además de y nunca en lugar de la metodología del pensamiento científico o el cuerpo de doctrina científica que nunca quedan anticuados. ¿Queremos formar máquinas capaces de apretar tornillos, que serán inútiles el día que se decida sustituir los tornillos por clavos, o personas capaces de comprender lo que es cada cosa y dominar cualquier tipo de herramienta en cada caso? Hay un proverbio que dice: "no me des peces, enséñame a pescar".

Es evidente que el alumno, carente lógicamente de formación, se sentirá más atraído por actividades de tipo lúdico y frívolo que por aquellas encaminadas a desarrollar su intelecto. Por ello, las prácticas serán siempre preferibles a la "pura y dura teoría", ya que ello permite obtener el título de licenciado a corto plazo sin mayores esfuerzos y sin plantearse cuáles serán sus intereses en un plazo de tiempo sólo ligeramente mayor, ya que su futuro les espera "a la vuelta de la esquina". Sólo tiene sentido hacer algo si ese "algo" se comprende, ya que el "hacer por hacer" o "hacer para pasar el rato" o para "cubrir el expediente" (tanto por parte del alumno como del profesor), es una farsa y una pérdida de tiempo y dinero.

En mi opinión, para poder "hacer" primero hay que "saber". En el plan de estudios deberemos, por tanto, dar prioridad a la sabiduría (o el conocimiento) frente a la práctica, ya que no se puede practicar nada en la ignorancia. Este mismo planteamiento es el que subyace en esa moda reciente y creciente que pretende conferir un matiz aplicado a los estudios de Biología (dicho sea de paso, lo mismo ha acontecido en lo referente a la investigación, quedando la investigación básica relegada a un segundo plano). Surge así otra posibilidad de error en nuestros planteamientos a la hora de elaborar un plan de estudios: la Ciencia (en este caso la Biología) aplicada debe predominar sobre la Ciencia pura. De este modo podemos 
apresurarnos a proponer asignaturas con magníficos titulares para atraer al alumno hacia lo práctico, lo aplicado y lo que supuestamente le va rendir beneficios económicos a corto plazo. La realidad es bien distinta, y el desencanto llegará cuando el alumno perciba que lo único que tiene de aplicado la asignatura está en el "rótulo". La verdad es que no puede ser de otra manera, ya que como dijo Pasteur "no existe ciencia pura y ciencia aplicada, existe la Ciencia y....las aplicaciones de la Ciencia.

\section{Perfil formativo}

El denominado perfil formativo básico de los planes de estudio en Biología no es, desgraciadamente, el más adecuado para la formación del futuro biólogo. La causa de ello es que en la elaboración de los planes y el establecimiento de cuales serían las disciplinas básicas que todo biólogo debe conocer, no se han seguido criterios científicos y, por lo tanto, la denominación de una asignatura como "troncal", "obligatoria" u "optativa" responde más bien a una lucha de intereses particulares, al capricho, la casualidad o la contingencia. El resultado es que cuando planteamos a nuestros recién licenciados cuestiones tales como ¿qué es la Biología? ¿qué es la vida? o ¿qué es la Biodiversidad? sus respuestas son tremendamente ambiguas y no pasan de simples balbuceos incoherentes.

Pero, a mi modo de ver, existen además causas de otra índole responsables también del desafortunado plan de estudios que actualmente profesamos. La Biología actual se encuentra enmarcada en una vía excesivamente reduccionista y cada vez es más frecuente encontrar profesores que no manifiestan su opinión sobre cuestiones que se salen de su estricto campo de hiperespecialización.

Creo que actualmente se abruma al alumno con numerosos datos, listas y protocolos que no son más que análisis particulares (y muchas veces parciales) de una 
determinada materia. Cada asignatura se convierte así en una solitaria y tediosa descripción.

Es obvio que el análisis y la descripción son absolutamente necesarios para poder abordar con garantías la enorme complejidad de los fenómenos biológicos, máxime cuando se trata de formar coherentemente a personas que carecen de las bases de conocimiento necesarias para enfrentarse a unos estudios universitarios. Sin embargo, una vez realizado ese análisis hace falta también enseñar al alumno a relacionar todos los datos. Hace falta entonces una síntesis; una síntesis que proporcione al alumno esa visión y comprensión global de todos los fenómenos biológicos y un marco de referencia donde situar todos los nuevos datos que la Ciencia de la Biología va constantemente produciendo.

Pienso que toda ciencia en la que el análisis no vaya acompañado de su correspondiente síntesis es una ciencia muerta, y el alumno será el primero en sufrir las consecuencias.

No hay ninguna duda de que todas las asignaturas y prácticas de la licenciatura de Biología por muy analíticas y particulares que sean, son intrínsecamente buenas y positivas. Sin embargo, todo biólogo (y todo científico y empresario) sabe hoy día que el "todo" no se explica con el mero sumatorio de las "partes". La Biología actual sabe que una molécula es más que la sola agregación de átomos. Que una célula es algo más que la simple agregación de moléculas. Que un organismo (pluricelular) no es sólo un simple conjunto de células (y tejidos, órganos...). Que una población es más que un mero agregado de organismos. Volviendo al símil del edificio, éste es más que el sumatorio de puertas, paredes, muebles etc.

Igualmente, un Plan de estudios tiene que ser algo más que la simple adición de asignaturas. Es probable que hasta ahora nos haya faltado esa visión global de lo que 
debe ser un edificio y nos hayamos peleado y zancadilleado por poner en él ese accesorio o complemento particular más grande que el de nuestro vecino.

No vendría mal reflexionar sobre estos aspectos si no queremos caer en los mismos errores a la hora de rehacer el perfil formativo del futuro Plan de estudios de Biología.

\section{Optatividad y libre elección}

Globalmente y de modo general, soy contrario a la excesiva optatividad en los planes de estudio de Biología. De todos modos dicha optatividad es sólo aparente, ya que en la práctica es todo lo contrario. Sin embargo ésta limitada optatividad no se debe, una vez más y por desgracia, a criterios científicos y docentes sino a una mera cuestión de horarios resultado, a su vez, del interés y el capricho como ya hemos apuntado anteriormente. Efectivamente, los horarios de las optativas suelen ser bastante incompatibles con los de las obligatorias y troncales, por lo que en realidad no hay tanta maniobra para optar. Esto implica que el alumno opte únicamente en base a lo que su apretado horario le permite y no por lo que verdaderamente tiene interés. De esta manera queda pervertido el espíritu de la optatividad.

Pero cabe plantearse la necesidad de la "libertad de elección", o lo que es lo mismo, curriculum abierto versus curriculum cerrado, plan regular o "plan a la carta".

Como se indicó al principio de este apartado, preferimos un plan cerrado con optatividad limitada. Volviendo al símil del edificio, éste debe construirse con orden, piso a piso, sin pretender poner los muros y las paredes antes que los cimientos o los muebles antes que los suelos. 
Creo que la elaboración de un "curriculum a la carta" que resulte coherente presupone una madurez en nuestros alumnos de la que claramente carecen, y de la que de igual modo nosotros carecíamos a su edad. En todo caso, esta situación les obliga a enfrentar sus intereses a corto plazo (aprobar cuanto antes y de la manera más rápida posible), con sus intereses a largo plazo (aprender una ciencia) por lo que deberíamos velar nosotros, los que ya somos adultos y maduros dentro de la Comunidad Académica. Un caso muy similar se daría si, por ejemplo, dejáramos elegir a nuestros hijos libremente su comida; lo más probable es que elijan caramelos, dulces, chocolate, etc.,. ¿No será más lógico obligarles a consumir frutas, verduras, proteínas, productos lácteos, etc. hasta que el paladar se acostumbre a ello y, entonces, hasta serán de su agrado además de imprescindibles para su correcto desarrollo?

En cuanto a las asignaturas de libre configuración, éstas plantean el problema de que pueden ser cursadas casi en cualquier año de la licenciatura, lo que provoca que el nivel de los alumnos en una misma clase sea muy heterogéneo y haya que partir siempre desde cero con la consiguiente repetición de contenidos y la pérdida de tiempo y nivel.

Por último, la libre elección no debería incluir otro tipo de actividades formativas fuera del ámbito de una determinada ciencia. Es magnífico que el alumno, además de Biología, desee tener conocimientos de guitarra por ejemplo, pero ello deberá aprenderlo extracurricularmente y por sus propios medios. Además, precisamente hoy día no faltan métodos, medios y lugares para obtener información de materias concretas.

\section{Especialidades}


En mi opinión las especialidades son convenientes dentro de la licenciatura de Biología. Pero sólo tres. A saber:

1.- Biología fundamental y funcional. Se ocuparía de los procesos vitales, de aquello que es común y general para todos los organismos vivos según el método científico convencional a base de análisis y algunas síntesis parciales.

2.- Biología sistemática y evolutiva. Se ocuparía del estudio de la diversidad biológica según el método convencional (o descriptivo) y representaría la parte analítica, y las causas de dicha diversidad, que representaría la primera gran síntesis biológica: la evolución o síntesis evolutiva según un método integrador y comparativo.

3.- Biología ecosistémica o Ecología. Se ocuparía del tratamiento descriptivo del funcionamiento de los ecosistemas, y representaría la parte analítica, y la segunda gran síntesis biológica: la integración de la diversidad biológica en los ecosistemas y la propia diversidad ecosistémica según un método integrador y comparativo.

El Plan de estudios de Biología debería huir del exceso de especialidades para evitar caer en la hiperespecialización más propia de un tercer ciclo.

Las actuales especialidades están tan claramente diferenciadas entre sí, que el alumno pierde de vista la unidad de la Biología. Frecuentemente los alumnos de una determinada especialidad me comentan con pena su falta de formación en otra especialidad en la que manifiestan también su interés.

\section{Horario}

El horario debería ser suficientemente flexible de manera que pueda permitir la recuperación de clases perdidas o de prácticas de campo que debido a la 
imprevisibilidad meteorológica no hayan podido realizarse el día que se había previsto con días o meses de antelación.

El gran número de asignaturas diversas e inconexas por cuatrimestre y la cantidad de datos suministrados en cada una de ellas, además de la gran cantidad de horas que el alumno pasa en clase, hace que éste no disponga de tiempo ni para comer, tal y como declaran en muchas ocasiones. Si no hay tiempo ni para comer, mucho menos habrá para estudiar, leer y reflexionar sobre lo aprendido. El resultado es que el alumno se aprende de memoria y se empapa de datos inconexos deprisa y corriendo para después "escupirlos" en el examen y olvidar rápidamente al día siguiente y liberarse así de una pesada carga. De esta manera carecen de los marcos de referencia donde ubicar adecuadamente sus conocimientos sobre Biología y comprender y dar sentido al complejo entramado de relaciones que establecen los seres vivos entre sí.

Por último, soy de la opinión de que la duración más adecuada para la licenciatura de Biología sería de 5 años. 


\begin{abstract}
Imagine sitting on the couch in your living room, at ten o'clock at night, quietly, in slippers, enjoying the privacy of your home and your favorite leisure activity, and it appeared a young, handsome, which, you kindly "the favor" to warn you Listen, hey, you you have the door open!.
\end{abstract}

I guess the shock would Morrocotudo. I'm not sure I appreciated the "favor". All I can imagine you would say borders on the edge of what is written here would reproduce rude.

\title{
KEY WORDS
}

Illegal access - Security - Privacy

Te imaginas sentado en el sofá del salón de tu casa, a las diez de la noche, tranquilamente, en zapatillas, disfrutando de la intimidad de tu hogar y de tu actividad de ocio preferida, y que apareciera un joven, bien parecido, que, muy amablemente te "hiciera el favor" de avisarte ¡Oiga, oiga, que tiene Vd. la puerta abierta!.

Supongo que el susto sería morrocotudo. No estoy muy segura de que agradecieras el "favor". Todo lo que puedo imaginar que dirías roza el límite de lo que reproducir por escrito aquí resultaría grosero.

Si en vez de decirte que la puerta está abierta, lo que te cuenta éste eficaz ciudadano es "Oiga, oiga, su puerta es blindada, y está cerrada, pero, debe Vd. saber qué, usando en conjunción, tales y tales herramientas, y siguiendo estas complejas 
instrucciones cualquiera es capaz de abrirla, a pesar de haberse $\mathrm{Vd}$. gastado tropocientas mil pesetas en la puerta. ¡fíjese, he tenido que ser yo el que venga a avisarle que no le sirve para nada!". ¿Qué tal lo ves ahora?. Quizá ahora me digas que violar tu intimidad es un delito y que podrías denunciarlo.

Puede, que además, el muy responsable y altruista sujeto te informe de que él no trabaja solo, que pertenece a un club de altruistas y responsables sujetos dispuestos a velar por la intimidad de la Sociedad, de forma completamente gratuita. Que son varios los que están, de forma coordinada, intentando descubrir las posibles maneras de entrar a las casas de los torpes incautos que no saben vigilar por si mismos de su seguridad. Te informa además de que ha difundido, entre todos los interesados, las instrucciones concretas para entrar a tu casa, eso sí, solo se lo ha dicho a personas que eran absolutamente de fiar, ya que se han identificado como pertenecientes a un club de altruistas salvaguardas de la intimidad.

¿Te quedas en casa a dormir?, ¿Pides permiso mañana en la oficina para dedicarte a seleccionar otra puerta blindada?. Quizá primero deberás pasar por el banco a pedir un préstamo. ¿Cuánto tiempo y dinero puedes o debes dedicar a proteger tu privacidad?.

Evidentemente ya sabes por donde voy. Voy a pedirte un esfuerzo mas, que donde hablábamos de tu casa nos refiramos a la información que reside en tu ordenador, donde hablábamos de puerta hablemos de mecanismos de seguridad, llamemos hacker al bien portado héroe que entró en tu casa y te arrancó de tu ignorancia ¡Mira que no saber que vivías inseguro!. Me estoy refiriendo a ese intruso que violó la intimidad de tu hogar.

Ahora te propongo que veamos la escena desde la otra perspectiva. Un conocido te cuenta que está viviendo una apasionante aventura, por el bien de la humanidad. Te 
cuenta que, todas las semanas, dedica horas interminables a ir puerta por puerta de la calle Mayor de tu ciudad, mientras los demás duermen, comprobando si están o no abiertas. Cuando una puerta está abierta, busca la llave debajo del felpudo, en defecto de la llave trata de abrirla con la ganzúa, en principio, intentando no causar daños materiales. Finalmente realiza un informe detallado de su actividad y se lo envía al propietario de la casa. ¿Qué concepto sacas del muchacho?. ¿Qué sentimientos te provocan su actividad? ¿Crees que el propietario de la vivienda se sentirá radiante de felicidad al leer el informe?.

En determinados entornos se pretende justificar la actividad de los hackers como bienhechores de una sociedad incauta que no sabe cuidar de si misma. Se insiste en que las empresas que han visto violada su intimidad deberían estar, encima, muy agradecidas por el desinteresado favor que el altruista caballero les ha hecho, viniendo, sin ser llamado, a resolverles la vida.

Parece que, hoy en día, hay bastante actividad en este sentido. Tengamos en cuenta que muchos de los accesos ilegales no son detectados, e incluso los detectados, frecuentemente, no son denunciados por las empresas, pues divulgar que se ha producido una entrada no autorizada puede suponer un descrédito para el buen nombre de la compañía. A pesar de ello, aparecen bastantes casos en la prensa.

Comentando estos temas con mi buen amigo Juan Manuel Morote, me decía: "que no, te equivocas ... que no es que los hackers utilicen una ganzúa para forzar mi puerta, a veces es que la vuelan a cañonazos".

A continuación, el inteligente y bien intencionado caballero puede aparecer en mi salón y decirme muy amablemente: "¡Oiga, oiga!, ¿Sabía que su puerta se puede volar con dinamita?". 